\title{
Relative mortality of Type 1 (insulin-dependent) diabetes in Denmark: 1933-1981
}

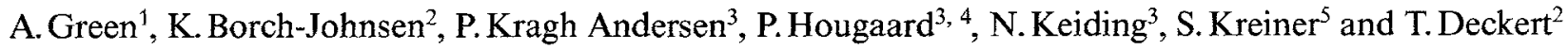 \\ ${ }^{1}$ University Institute of Clinical Genetics, Odense, ${ }^{2}$ Steno Memorial Hospital, Gentofte, ${ }^{3}$ Statistical Research Unit, Danish Medical and Social \\ Science Research Councils and University of Copenhagen, ${ }^{4}$ Institute of Mathematical Statistics, University of Copenhagen, Copenhagen and \\ ${ }^{5}$ Department for Data Processing, Copenhagen County Hospital, Herlev, Denmark
}

\begin{abstract}
Summary. The relative mortality of Type 1 (insulin-dependent) diabetes in Denmark during the period 1933-1981 was studied using a modification of Cox's regression model on the basis of two patient populations, ascertained in different ways and independently of each other. Initial analysis showed that the two groups could be combined completely into one common analysis. Relative mortality was the same for both sexes. The additional variables studied were age at diagnosis, current age, calendar year at diagnosis and calendar time during
\end{abstract}

follow-up. All these interrelated variables were accounted for in the analysis. The analysis showed that relative mortality (a) decreased with increasing age at diagnosis; (b) increased from 1933 to a maximum in about 1965 , after which it decreased; (c) increased with increased duration of diabetes to a maximum at 15-25 years, after which it declined.

Key words: Type 1 diabetes, mortality, regression analysis.
The introduction of insulin treatment by Banting and Best in 1922 [1] dramatically improved the prognosis of Type 1 (insulin-dependent) diabetes mellitus. However, long-term follow-up studies of patients treated with insulin since diagnosis have consistently demonstrated an excess mortality, so that the life expectancy for these patients is only about two-thirds of that for normal subjects [2-4].

It is well-known that this excess mortality is clinically associated with the development of renal and cardiovascular complications $[5,6]$. Among the epidemiologically identified indicators of the diabetes mortality are low age at the diagnosis of disease, high insulin dosage, lack of attendance at a diabetes clinic, and calendar year of diagnosis $[3,7]$. In addition to these factors, sex, the calender time during which the patients were followed and current age must be considered. This is particularly necessary when studying patients, diagnosed at different ages and at different calendar times, during long periods. Thus, analysis of diabetes mortality is complicated, which may explain why relatively little is know about the basic causes of the excess mortality.

The present report presents a statistical analysis of the relative mortality of Type 1 diabetes in Denmark during the period 1933-1981, with particular emphasis on the evaluation of possible secular trends and of effects of some previously reported indicators of the diabetes mortality. The study is based on a combined anal- ysis of two patients populations, ascertained in different ways and independently of each other, but both judged to represent Danish patients with Type 1 diabetes.

\section{Materials and methods}

\section{The patient populations}

Two different groups of diabetic patients were subject to analysis:

The Fyn County patient group. Using insulin prescriptions from a 5-month period, the prevalence population of insulin-treated diabetic patients in the Fyn County, Denmark, as of 1 July 1973, was ascertained as described previously [8]. The verification of diagnosis and the collection of supplementary clinical and personal data have also been described $[6,8,9]$. In the total patient group of 1499 subjects, judged to be $>98 \%$ complete and representative of the total Danish population of insulin-treated diabetic patients [8], all individuals were selected (429 males and 320 females) whose diagnosis was established before the age of 31 years.

The Steno Memorial Hospital patient group. The Steno Memorial Hospital in Greater Copenhagen was established in 1933 and has since then served as a diabetes specialist hospital for patients from the whole of Denmark. Patients are referred to the Steno from general practitioners and/or other hospitals. About two-thirds of the patients return to the referring physician or local hospital for continued control, while the remainder attend the outpatient clinic at the Steno for longer periods i. e. more than seven visits within the first 20 years after diagnosis. From the medical records at the Steno all patients (796 
Table 1. Clinical characteristics of the patient groups studied

\begin{tabular}{|c|c|c|}
\hline & $\begin{array}{l}\text { Fyn County } \\
\text { patients } \\
(n=749)\end{array}$ & $\begin{array}{l}\text { Steno } \\
\text { Memorial } \\
\text { Hospital } \\
\text { patients } \\
(n=1444)\end{array}$ \\
\hline \multicolumn{3}{|l|}{ No. of patients } \\
\hline Male & 429 & 796 \\
\hline Female & 320 & 648 \\
\hline Observation period & July 1973-1981 & 1933-1981 \\
\hline $\begin{array}{l}\text { No. of deaths recorded } \\
\text { during observation period }\end{array}$ & 111 & 829 \\
\hline \multicolumn{3}{|l|}{ At diagnosis: } \\
\hline No. aged $0-10$ years & $266(36 \%)$ & $518(36 \%)$ \\
\hline No. aged $11-20$ years & $266(36 \%)$ & $547(38 \%)$ \\
\hline No. aged $21-30$ years & $217(29 \%)$ & $379(26 \%)$ \\
\hline Median age (years) & 14 & 13 \\
\hline \multicolumn{3}{|l|}{ At entry into the study: } \\
\hline No. aged $0-20$ years & $178(24 \%)$ & $581(40 \%)$ \\
\hline No. aged $21-40$ years & $369(49 \%)$ & $686(48 \%)$ \\
\hline No. aged $41-60$ years & $173(23 \%)$ & $172(12 \%)$ \\
\hline No. aged $>60$ years & $29(4 \%)$ & $5(0.3 \%)$ \\
\hline Median age (years) & 32 & 25 \\
\hline
\end{tabular}

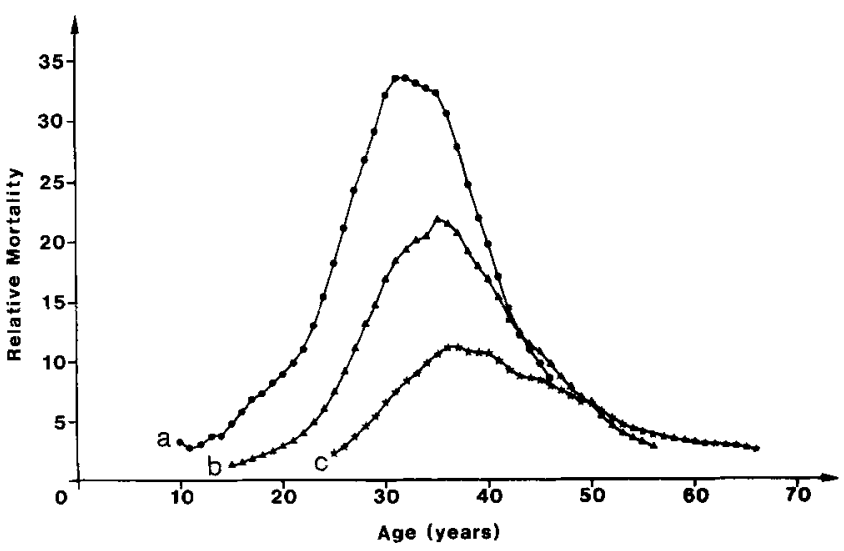

Fig.1. Relative mortality as a function of current age and selected levels of age at diagnosis. (a) (-) : age at diagnosis = 5 years; (b) $(\Delta-\Lambda)$ : age at diagnosis = 15 years; (c) ( $\mathcal{H}-\mathcal{H}$ ): age at diagnosis $=25$ years. Calendar year at diagnosis fixed at 1940

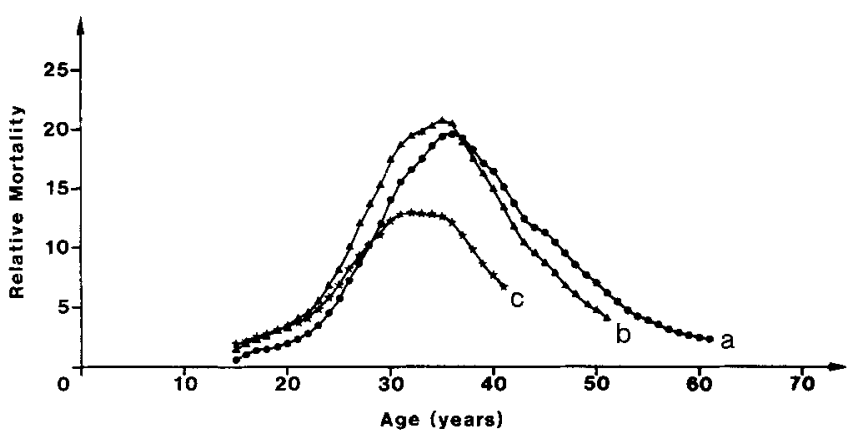

Fig. 2. Relative mortality as a function of current age and selected calendar years at diagnosis. (a) $(-$ ): year at diagnosis $=1935$; (b) $(\Delta-\Delta)$ : year at diagnosis $=1945$; (c) $(*-*)$ : year at diagnosis $=1955$. Age at diagnosis fixed at 15 years males and 648 females) whose Type 1 diabetes was diagnosed before age 31 years and before 1953, and who were admitted to the Steno for the first time before 1975 , were included in the study.

\section{Follow-up of the patients}

For all patients in both groups survival status was assessed as of $1 \mathrm{Jan}-$ uary 1982 from local public registries and/or the Danish centralized person registry, as described previously $[3,6]$. For deceased patients and emigrants, the date of death or emigration was recorded.

\section{Mortality data for the general population}

Mortality rates for the Danish population, specified according to age, sex and calendar time within the period 1933-1981, were obtained directly from Danmarks Statistik.

\section{Statistical analysis}

As the present study covers a long time (1933-1981) during which considerable changes in mortality in the general population took place, we decided to study diabetes mortality relative to population mortality, rather than in absolute terms. To this purpose a modification of Cox's regression model for survival data [10] was developed and applied here. In our model the covariates $z_{1}(a), \ldots, z_{k}(a)$ are assumed to have a multiplicative effect on relative mortality. The general formulation of the model is

$\lambda(\mathrm{a}, \mathrm{z})=\mu_{\mathrm{s}}(\mathrm{a}, \mathrm{t}) v_{0}(\mathrm{a}) \exp \left(\beta_{1} \mathrm{z}_{1}(\mathrm{a})+\ldots+\beta_{\mathrm{k}} \mathrm{z}_{\mathrm{k}}(\mathrm{a})\right)$

where $\lambda(a, z)$ is the death intensity (or hazard or force of mortality) at age a for a diabetic patient, with covariates $z_{1}(a), \ldots, z_{k}(a)$. The covariates include sex (s) and age at diagnosis of diabetes; $\mu_{s}(a, t)$ is the death intensity in the general population for a person of sex $\mathrm{s}$ and current age a at calendar time $t ; \beta_{1}, \ldots, \beta_{\mathrm{k}}$ are unknown regression coefficients to be estimated, and $v_{0}$ (a) represents the age-specific base-line relative mortality, i.e. the relative mortality for a patient of age a for whom $z_{1}(a)=\ldots=z_{k}(a)=0$.

In addition to sex and age at diagnosis, calendar year at diagnosis and the calendar time during which the patients were followed have been included as covariates. Because of the close interrelationship between the time-related covariates (for example, [calendar year at diagnosis] and [age at diagnosis] tie together [current age] and [follow-up period]), it is difficult to judge their effects independently. We therefore modelled these effects in a flexible way by means of stepwise constant functions, or quadratic or cubic polynomials, and evaluated their simultaneous influence by keeping some of the variables fixed and studying how the relative mortality varied as a function of the remaining variables.

The patients were included in the 'risk set' from age at entry into the study (from first admission to the Steno for the Steno patients, and from 1 July 1973 for the Fyn County patients) until age at death, emigration, or 1 January 1982, respectively.

The regression coefficients $\beta_{1}, \ldots, \beta_{\mathrm{k}}$ were estimated by maximizing the partial likelihood function [10], and $v_{0}$ was estimated by smoothing the Breslow-estimate of the cumulative base-line relative mortality as suggested by Ramlau-Hansen [11]. Hypotheses concerning the $\beta$ 's were tested using the partial likelihood ratio test-statistic $-2 \log Q$, and the assumption of proportional relative mortalities in the model was checked by means of the graphical methods and the teststatistic $Q_{1}$, described by Andersen [12].

\section{Results}

Clinical characteristics of the two patient groups are given in Table 1. As a consequence of the different ascertainment procedures and delineation criteria em- 
Table 2. Estimated values of relative mortality for selected values of age at diagnosis, current age and calendar time during follow-up

\begin{tabular}{|c|c|c|c|c|c|c|}
\hline \multirow{2}{*}{$\begin{array}{l}\text { Age at } \\
\text { diagnosis } \\
\text { (years) }\end{array}$} & \multirow{2}{*}{$\begin{array}{l}\text { Current } \\
\text { age } \\
\text { (years) }\end{array}$} & \multicolumn{5}{|c|}{$\begin{array}{l}\text { Relative Mortality by Calendar year at ob- } \\
\text { servation }\end{array}$} \\
\hline & & 1938 & 1948 & 1958 & 1968 & 1978 \\
\hline \multirow[t]{3}{*}{10} & 25 & 3.8 & 9.1 & 12.5 & 9.9 & 4.5 \\
\hline & 35 & - & 14.6 & 25.6 & 26.0 & 15.2 \\
\hline & 45 & - & - & 9.6 & 12.4 & 11.5 \\
\hline \multirow[t]{3}{*}{20} & 35 & 4.9 & 11.9 & 16.4 & 13.0 & 5.9 \\
\hline & 45 & - & 5.3 & 9.4 & 9.5 & 5.5 \\
\hline & 55 & - & - & 3.4 & 4.5 & 3.3 \\
\hline \multirow[t]{3}{*}{30} & 45 & 2.2 & 5.2 & 7.2 & 5.7 & 2.6 \\
\hline & 55 & - & 2.3 & 4.0 & 4.1 & 2.4 \\
\hline & 65 & - & - & 3.0 & 3.9 & 2.9 \\
\hline
\end{tabular}

ployed, all information regarding the mortality pattern before 1973 originates from the observation of the Steno patient group, which is also characterized by showing a younger median age at entry into the study. On the other hand, all information regarding the mortality pattern among patients diagnosed after 1952 originates from the Fyn County patients.

The study groups were initially analysed separately. Since neither the individual regression coefficients nor the entire sets of coefficients from the two groups differed significantly $(-2 \log \mathrm{Q}=6.18,8 \mathrm{df}, p=0.63)$, the model was reduced to one with common coefficients for the groups, but with different base-line relative mortalities. To reduce the model further, it was tested to see whether these base-line relative mortalities could be considered first proportional and, finally, identical. Since neither hypothesis could be rejected $\left(\mathrm{Q}_{1}=8.85\right.$, $5 \mathrm{df}, p=0.12$; and $-2 \log \mathrm{Q}=1.92,1 \mathrm{df}, p=0.17$, respectively), the two patient groups were combined in a common model. In this model the relative mortality was slightly lower for male than for female patients (the maximum partial likelihood estimate for males, $\hat{\beta}_{\text {male }}=-0.041 ; \beta_{\text {female }}$ set to zero). However, since this effect of sex was small and far from statistical significance $(p=0.54)$, sex was excluded from the model.

The remaining covariates in the final model are all related to time. Stepwise checking of the model assumptions during the analysis demonstrated that the effect of age at diagnosis depended on ("interacted with") the current age ( $p$ for interaction: <0.001). In addition to the effect of age at diagnosis, both calendar year at diagnosis and calendar time during follow-up $(p<0.0001)$ had to be accounted for.

An overall evaluation of the results is best done by examination of the values of relative mortality corresponding to selected covariate values.

Age at diagnosis combined with current age; fixed calendar year at diagnosis. The relative mortality decreased with increasing age at diagnosis (or, equivalently, with decreasing duration of disease), for values of current age less than approximately 40 years. For higher values of current age there was no effect of age at diagnosis. This trend is illustrated in Figure 1 for selected ages at diagnosis and with 1940 as year at diagnosis.

Calendar year at diagnosis combined with current age; fixed age at diagnosis. The relative mortality increased, for values of current age less than 35 years, as calendar year at diagnosis increased up to approximately 1945. As calendar year at diagnosis increased after 1950, the relative mortality decreased. This trend is illustrated in Figure 2 for selected calendar years at diagnosis and with age at diagnosis fixed at 15 years.

Calendar time during follow-up. For fixed values of the other covariates, there was a generally increasing relative mortality for calendar time during follow-up until the mid-1960s, after which time the relative mortality declined considerably. Examples are given in Table 2, which also illustrates in further detail the trends described above.

When the covariates were accounted for, the estimated relative mortality had a characteristic form (Figs. 1 and 2) with a maximum corresponding to values of current age between 30 and 40 years (or duration of diabetes between 15 and 25 years), after which age it declined towards a level of approximately 2 at age 55 years (or after 40-50 years of diabetes).

\section{Discussion}

Both patient groups in our study have previously been judged representative of Danish diabetic patients, and since only insulin-treated patients with diagnosis established before age 31 years were included we believe that our results give a valid picture of mortality in Type 1 diabetes in Denmark. Because of secular changes in the mortality pattern in the general population, we decided to study and present estimates of the relative mortality, but absolute mortality rates can be estimated by multiplication of the values in Table 2 with the relevant age-, sex- and calendar time-specific rates for the general population (equation $\left({ }^{*}\right)$ ). Alternatively, we could have attempted to analyse the absolute mortality for the patients directly, in a regression model including calendar time as a covariate. However, such an approach would not have produced a description, common for males and females, as simple as that given above.

Our analysis has shown that several time-related variables must be simultaneously accounted for in the description of the relative mortality of Type 1 diabetes. Due to the mutual relationship between these variables, separate evaluation of each of them is difficult. However, when their effects are combined with the underlying (base-line) relative mortality in estimates for various levels of the variables, certain characteristic features appear. 
First, it has been confirmed that Type 1 diabetes is associated with a considerable relative mortality. Studies of the absolute mortality have shown a higher mortality in males than in females [3,6], but our analysis of the relative mortality indicates that the impact of Type 1 diabetes regarding mortality is the same for both sexes.

The secular trend in relative mortality with an increase up to about 1960 is surprising. One would have expected an opposite trend because of the gradual improvement in diabetes care since the introduction of insulin. Even so the period 1933-1960 was characterized by a steady decrease in population mortality which might not necessarily influence the absolute mortality among diabetic patients. Also, the maximum at about 1960 coincides with the finding of an increasing incidence of nephropathy among patients with Type 1 diabetes from 1940 to 1960 , with a mean survival time of 7 years after the onset of disease [5]. The trend could also be at least partly explained by secular trends in the clinical features of patients referred to the Steno, but this seems unlikely since it has been shown previously that Steno patients do not differ from patients from population-based Danish studies with respect to certain clinical characteristics [13]. Regardless of these aspects, our study clearly demonstrates that relative mortality of Type 1 diabetes has decreased considerably during the last 20 years, maybe as a result of efforts to improve diabetes care.

The present study also confirms that onset of diabetes early in life is associated with increased relative mortality. However, because of the interaction between current age, age at diagnosis, and duration of diabetes, we cannot directly determine the effect of age at diagnosis. The maximal relative mortality at age $30-40$ years corresponds with the finding that diabetic patients in this age group have the highest proportion of deaths due to renal complications [6,14]. The associations between relative mortality and age at diagnosis (or duration of diabetes) and current age could be explained by the hypothesis that Type 1 diabetes involves a progressive pathological process leading to the development of complications, but that certain patients escape the fatal complications, such as nephropathy so that, after 20-25 years the relative mortality gradually decreases. If correct, this hypothesis has important implications regarding the identification of measures that can prevent or at least decelerate this process.

Finally, our study has shown that many mutually interrelated factors determine the mortality in Type 1 diabetes. Therefore, analysis of the mortality of diabetes is a complicated task that requires the use of advanced statistical methodology. However, we find it important and encouraging that the use of such techniques makes it possible to combine two patient groups ascertained in completely different ways into one common analysis, thereby maximizing the utilization of information in each of the groups. We recommed that a similar methodological approach be applied to the analysis of mortality and morbidity of Type 1 diabetes in other well-defined patient populations, so as to improve our understanding of the causes and potential for reduction of the complications and mortality associated with this disease.

Acknowledgements. This study was supported by The Danish Medical Research Council (grant no.12-3431) and from Dr. med. Erik Garde og Elisabeth Gardes Legat.

\section{References}

1. Banting FG, Best CB, Collip JB, Cambell WR, Fletcher AA (1922) Pancreatic extract in the treatment of diabetes mellitus. Canad Med Ass J 2: 141-146

2. Marks HH (1965) Longevity and mortality of diabetes. Am J Publ Health 55: 416-423

3. Deckert T, Poulsen JE, Larsen M (1978) Prognosis of diabetics with diabetes onset before the age of thirty-one. Diabetologia 14: 363-377

4. Goodkin G (1975) Mortality factors in diabetes. J Occup Med 17: 716-721

5. Andersen AR, Christiansen JS, Andersen JK, Kreiner S, Deckert T (1983) Diabetic nephropathy in type 1 (insulin-dependent) diabetes: an epidemiological study. Diabetologia 25:496-501

6. Green A, Hougaard P (1984) Epidemiological studies of diabetes mellitus in Denmark: 5 . Mortality and causes of death among insulin-treated diabetic patients. Diabetologia 26: 190-194

7. Dorman JS, LaPorte RE, Kuller LH, Cruickshanks BA, Orchard TJ, Wagener DK, Becker DJ, Cavender DE, Drash AL (1984) The Pittsburgh insulin-dependent diabetes mellitus (IDDM) morbidity and mortality study. Mortality results. Diabetes 33: 271-276

8. Green A, Hauge M, Holm NV, Rasch LL (1981) Epidemiological studies of diabetes mellitus in Denmark: II. A prevalence study based on insulin prescriptions. Diabetologia 20: 468-470

9. Green A, Hougaard P (1983) Epidemiological studies of diabetes mellitus in Denmark: 4. Clinical characteristics of insulin-treated diabetes. Diabetologia 25: 231-234

10. Kalbfleisch JD, Prentice RL (1980) The statistical analysis of failure time data. Wiley, New York

11. Ramlau-Hansen $H$ (1983) Smoothing counting process intensities by means of kernel functions. Ann Statist 11: 453-466

12. Andersen PK (1982) Testing goodness of fit of Cox's regression and life model. Biometrics 38: 67-77

13. Deckert T, Poulsen JE, Larsen M (1979) The prognosis of insulindependent diabetes mellitus and the importance of supervision. Acta Med Scand (Suppl) 624: 48-53

14. Moloney A, Tunbridge WMG, Ireland JT, Watkins PJ (1983) Mortality from diabetic nephropathy in the United Kingdom. Diabetologia $25: 26-30$

Received: 19 September 1984 and in revised form: 15 May 1985

Dr. Anders Green

Institute of Clinical Genetics

J. B. Winsløwsvej 17

DK-5000 Odense C

Denmark 